\title{
Experiencia de un Ciclo de Mejora en el Aula en el ámbito de la Enfermería del Envejecimiento durante la COVID 19
}

\section{Experience of an Improvement Cycle in Classroom in the field of Geriatric Nursing during COVID 19}

ROCío ROMERO SERRANO

ORCID: https://orcid.org/0000-0002-2973-4919

Centro Universitario de Enfermería

San Juan de Dios.

Adscrito a la Universidad de Sevilla.

rocio.romero@sjd.es

DOI: http://dx.doi.org/10.12795/9788447231003.061

Pp.: 1294-1312 


\section{Descripción del contexto de intervención}

El Ciclo de Mejora en el Aula (CIMA) que se presenta, se ha realizado en 3o curso del Grado en Enfermería, en la asignatura Enfermería del Envejecimiento. Es obligatoria y tiene 6 créditos ECTS. El grupo estaba formado por 77 estudiantes, el $75,3 \%$ son mujeres y el $24,7 \%$ son hombres.

El CIMA se ha aplicado en 8 horas. Se ha trabajado contenido conceptual, procedimental y actitudinal, relacionado con el envejecimiento. Se han realizado 6 horas en grupo grande, y las 2 horas restantes en grupos pequeños (la clase para esta metodología se ha dividido en 4 grupos con una media de 20 estudiantes por clase). Estas 2 horas finalmente se hicieron en grupo pequeño pero online, siguiendo las indicaciones por la Pandemia COVID 19.

La finalidad de los contenidos teóricos de esta asignatura pretenden que los estudiantes alcancen los siguientes objetivos:

- Describir y comprender los cambios asociados al proceso de envejecer y su repercusión en la salud.

- Identificar las modificaciones estructurales, funcionales, psicológicas y de formas de vida asociadas al proceso de envejecer.

- Seleccionarlas intervenciones cuidadoras dirigidas a tratar o a prevenir los problemas de salud y su adaptación a la vida diaria

A ello hay que unir las competencias trasversales con las que pretendemos que adquieran la capacidad de aplicar los conocimientos en la práctica, capacidad para trabajar en un equipo interdisciplinar, capacidad de aprender y toma de decisiones.

Ciclos de Mejora en el Aula (2020). Experiencias de Innovación Docente de la US Esta obra se distribuye con la licencia Creative Commons 
Esto es lo que se describe en el programa de la asignatura, pero como profesora y conocedora de las necesidades de mis estudiantes, me planteaba un cambio como docente. Además, en esta línea de innovación conocía el trabajo de otras colegas, como Dolores Guerra (2017) y Rocío de Diego (2020). Entonces, decidí realizar el Curso General De Docencia del Programa De Formación e Innovación Docente Del Profesorado de la Universidad de Sevilla y fue donde realicé lo que se conoce como CIMA.

\section{Diseño del CIMA}

En el desarrollo de este CIMA se han tenido en cuenta las aportaciones y experiencia vivida en el Curso General de Docencia Universitaria de la Universidad de Sevilla.

En esta sección se describe el diseño el CIMA, realizado antes de empezar su aplicación. Se incluye el mapa de contenido, el modelo metodológico, la secuencia de actividades diseñadas y el cuestionario que realizó el alumnado antes y después del CIMA.

\section{Mapa de contenidos y problemas}

Recientemente ha sido publicado por el Consejo Superior de Investigaciones Científicas (CSIC) un artículo en el que se afirma que en España sigue creciendo en mayor medida la proporción de personas mayores de ochenta años (octogenarios/as),el 6,1\% de toda la población (Pérez, Abellán, Aceituno y Ramiro, 2020). Además, la proyección del Instituto Nacional de Estadística (INE) para el 2066 es que habrá más de 14 millones de personas mayores, con edad mayor o igual a 65 años, eso significa el 34.6\% del total de la población. Nos encontramos con el fenómeno denominado envejecimiento del envejecimiento (Úbeda, 2009). Con estos motivos ya podríamos justificar la necesidad de 
una asignatura centrada en las personas mayores, pero es que además nos encontramos con una población mayor pluripatológica y con enfermedades crónicas.

Por ello, debemos explicar que las personas mayores tienen unas características propias en la forma de aparición de las enfermedades y en sus repercusiones funcionales, psíquicas y sociales. Y para evaluarlas se utilizan instrumentos que cumplen requisitos de validez, fiabilidad, factibilidad, sencillez y brevedad de aplicación. Por todo ello, se plantea la Valoración Geriátrica Integral (VGI) como el método para medir la capacidad funcional, psíquica y social, siendo interdisciplinar, ya que participan profesionales de distintas disciplinas (Rodríguez, Zas, Silva, Sanchoyerto y Cervantes, 2014). En este abordaje interdisciplinar de la persona mayor, la contribución explícita de las enfermeras está avalada por el uso de un modelo conceptual y la aplicación del proceso enfermero a la práctica clínica (Vallejo, Rodríguez \& Valverde, 2007).

Ahora bien, desde un planteamiento docente creemos que para la adquisición de estos conocimientos es necesario un trabajo colaborativo para la resolución de los problemas, promoviendo un equilibrio entre la teoría y la práctica (Tourón y Santiago, 2015). Así que, se evitará la clase tradicional, fomentando el aprendizaje reflexivo e investigativo (Gureckis y Markant, 2012).

Se elaboró un mapa de contenidos (Figura 1), ya que se considera una herramienta de apoyo para organizar los contenidos más relevantes (García, Porlán y Navarro, 2017). En él se exponen las preguntas clave y los contenidos conceptuales, procedimentales y actitudinales que han guiado la actuación docente.

Ciclos de Mejora en el Aula (2020). Experiencias de Innovación Docente de la US Esta obra se distribuye con la licencia Creative Commons 


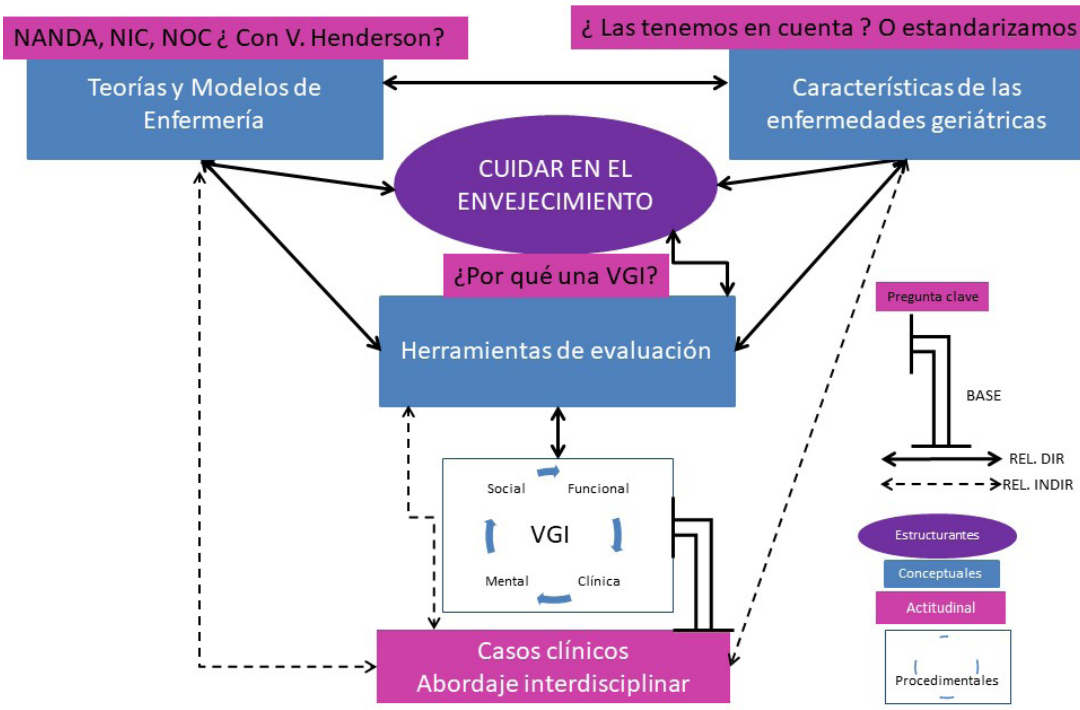

Figura 1. Mapa de contenidos

\section{Modelo metodológico y secuencia de actividades}

El modelo metodológico planteado para este CIMA (Figura 2) está basado en mi experiencia previa como docente, junto con todo lo aprendido en la realización del Curso General de Docencia Universitaria (Universidad de Sevilla). Esta asignatura, tal y como se refleja en su Proyecto Docente, tiene 38 horas de clases teóricas y 22 horas en clases en seminarios (Romero, 2020).

En las clases teóricas se suele seguir un modelo basado en la transmisión, es decir todo el protagonismo es del docente. Con el nuevo modelo metodológico planteado se adopta un enfoque basado en la reelaboración de las ideas de los estudiantes, en el cual se aboga por un aprendizaje que resulte de la interacción entre los conocimientos que tienen los estudiantes en su mente, y la información que la docente les hace llegar proveniente de

Ciclos de Mejora en el Aula (2020). Experiencias de Innovación Docente de la US Esta obra se distribuye con la licencia Creative Commons 
fuentes diversas (Porlán, 2017). Para ello, hemos partido de los conocimientos previos que el alumnado tiene, pasándole un cuestionario inicial, y tras analizarlo, se ha realizado una secuencia de actividades.

En el modelo metodológico planteado, podemos observar que comenzamos con una breve introducción (a modo de presentación del esquema que va a llevar la clase). A continuación, realizamos al alumnado una pregunta que les conecte, que les active su interés, para a continuación plantearles un problema (una pregunta a resolver, un caso clínico que resolver). Para ello la profesora hace una exposición, que sería como la clase magistral que se daba, pero en lugar de ocupar dos horas, se plantea en 30-40 minutos junto con una actividad de contraste; por ejemplo, análisis de artículos y sacar las ideas principales. Finalmente, tanto ellos como la profesoraexponen las conclusiones más relevantes y da por finalizada la ann

Introducción (I)

Actividad de contraste (Ac)

Expresión de Ideas del alumnado (le)

Exposición de conceptos (E)

Problemas $(\mathrm{Pr})$

Conclusiones (C)

Figura 2. Modelo Metodológico

En la siguiente tabla se presenta la secuencia de actividades diseñadas, tiempo empleado, recursos y tipos de contenidos trabajados (conceptuales, procedimentales y actitudinales) en cada una de las sesiones (Tabla 1).

Ciclos de Mejora en el Aula (2020). Experiencias de Innovación Docente de la US Esta obra se distribuye con la licencia Creative Commons 
ROcío ROMERO SERRANO

Tabla 1. Secuencia de actividades

\begin{tabular}{|l|l|l|l|l|}
\hline N.o & Actores & Contenido & Descripción \\
\hline 1 & Profesora & $\begin{array}{l}\text { Presentación CIMA. } \\
\text { Que el alumnado conozca el objetivo de la } \\
\text { realización de las actividades }\end{array}$ & Presentación CIMA & $10^{-}$ \\
\hline 2 & Alumnado & $\begin{array}{l}\text { Cuestionario inicial. } \\
\text { Diseñar la secuencia de actividades }\end{array}$ & Realización del cuestionario \\
\hline 3 & $\begin{array}{l}\text { Alumnado } \\
\text { Profesora }\end{array}$ & $\begin{array}{l}\text { Collage de personas mayores (La profesora } \\
\text { presenta un collage con “diferentes tipos de } \\
\text { personas mayores). } \\
\text { Conocer las caracteristicas del } \\
\text { envejecimiento funcional, mental (afectivo y y } \\
\text { cognitivo) y social. }\end{array}$ & $\begin{array}{l}\text { Se presenta el collage } \\
\text { Se realiza lluvia de ideas y posterior debate } \\
\text { de qué ven/ identifican en esas personas } \\
\text { mayores. } \\
\text { Debate sobre las características que ellos } \\
\text { han comentado y las que hemos visto en los } \\
\text { cuestionarios. } \\
\text { Búsquedas en la web de art con expliquen el } \\
\text { envejecimiento y presentación en clase. } \\
\text { Exposición de las caracteristicas el } \\
\text { envejecimiento fisiológico: físico, mental y } \\
\text { social (Contenido conceptual) }\end{array}$ & 20- \\
\hline
\end{tabular}

Ciclos de Mejora en el Aula (2020). Experiencias de Innovación Docente de la US

cc (i) $\odot$ Esta obra se distribuye con la licencia Creative Commons 
ROCío ROMERO SERRANO

\begin{tabular}{|c|c|c|c|c|}
\hline 4 & $\begin{array}{l}\text { Alumnado } \\
\text { Profesora }\end{array}$ & $\begin{array}{l}\text { Herramientas: } \\
\text { Qué conocéis y qué podemos hacer. } \\
\text { (Basándonos en las fotos del collage). } \\
\text { Aplicar a personas mayores las herramientas } \\
\text { de evaluación más importantes. }\end{array}$ & $\begin{array}{l}\text { Búsquedas en páginas oficiales de la } \\
\text { aplicación de diferentes cuestionarios/ } \\
\text { escalas/test y presentarlas. } \\
\text { Analizar las distintas herramientas de } \\
\text { evaluación en personas mayores "diferentes" } \\
\text { Basada en las fotos del Collage. } \\
\text { Conclusiones. } \\
\text { (Contenido procedimental) }\end{array}$ & $\begin{array}{l}20^{\prime} \\
30^{-} \\
20^{-}\end{array}$ \\
\hline 5 & $\begin{array}{l}\text { Profesora } \\
\text { Alumnado }\end{array}$ & $\begin{array}{l}\text { Características de las enfermedades } \\
\text { geriátricas } \\
\text { (Basándonos en las fotos del collage). } \\
\text { Utilizar las herramientas en la particularidad } \\
\text { sintomática de las personas mayores. }\end{array}$ & $\begin{array}{l}\text { Se presentan las características que ellos } \\
\text { han descrito en el cuestionario. } \\
\text { Se relacionan con las fotos del collage. Ellos } \\
\text { deben explicar qué interrelación ven } \\
\text { Se explican las características de las } \\
\text { enfermedades geriátricas. } \\
\text { Búsqueda de artículo. Ultimas evidencias del } \\
\text { envejecimiento fisiológico } \\
\text { Conclusiones. } \\
\text { (Contenido conceptual) }\end{array}$ & $\begin{array}{l}10^{-} \\
20^{-} \\
40^{\circ} \\
20^{\circ} \\
20^{-}\end{array}$ \\
\hline
\end{tabular}

Ciclos de Mejora en el Aula (2020). Experiencias de Innovación Docente de la US cc (7) $(2)$ Esta obra se distribuye con la licencia Creative Commons BY NC ND 


\begin{tabular}{|c|c|c|c|c|}
\hline 6 & Profesora & $\begin{array}{l}\text { Modelo sí e interdisciplinariedad también. } \\
\text { Conocer los modelos de Enfermería y su } \\
\text { interconexión con las herramientas. } \\
\text { Conocer las características del trabajo } \\
\text { en equipo (interdisciplinariedad/ } \\
\text { multidisciplinariedad). }\end{array}$ & $\begin{array}{l}\text { Lluvia de ideas, qué modelos conocéis } \\
\text { Se presentan los modelos más utilizados en } \\
\text { enfermería. } \\
\text { El alumnado expondrá sus vivencias en } \\
\text { las prácticas de centro residenciales y } \\
\text { si observaron/les explicaron desde qué } \\
\text { modelo de Enfermería. } \\
\text { Conocer la complejidad del adulto mayor y la } \\
\text { pertinencia de la asistencia multidisciplinar. } \\
\text { En el caso de enfermería desde la aplicación } \\
\text { de un modelo. } \\
\text { Casos de asistencia multi/interdisciplinar. } \\
\text { Análisis de art. } \\
\text { Conclusiones } \\
\text { (Contenido procedimental y conceptual) }\end{array}$ & $\begin{array}{l}10^{-} \\
30^{-} \\
20^{-} \\
20^{-} \\
20^{-} \\
10^{-}\end{array}$ \\
\hline 7 & $\begin{array}{l}\text { Profesora } \\
\text { Alumnado } \\
\text { en grupos } \\
\text { pequeños }\end{array}$ & $\begin{array}{l}\text { Casos clínicos. } \\
\text { Aplicación y análisis de la VGI. Puesta en } \\
\text { común de los subgrupos. }\end{array}$ & $\begin{array}{l}\text { Presentación de puntos clave en la } \\
\text { realización de VGI } \\
\text { Se realizarán en grupos de } 3 \text { o } 4 \text { estudiantes } \\
\text { casos reales, se presentarán en clase y } \\
\text { se resaltarán los puntos clave que deben } \\
\text { hacerse siempre y se perfilarán las posibles } \\
\text { áreas de mejora si es que las hubiera. } \\
\text { Conclusiones } \\
\text { (Contenido conceptual, procedimental y } \\
\text { actitudinal). }\end{array}$ & $\begin{array}{l}20^{-} \\
50^{-} \\
10^{-}\end{array}$ \\
\hline 8 & Alumnado & Cuestionario final & El alumnado realizará el cuestionario & $30^{-}$ \\
\hline
\end{tabular}

Ciclos de Mejora en el Aula (2020). Experiencias de Innovación Docente de la US cc (7) $($ Esta obra se distribuye con la licencia Creative Commons BY NO ND 


\section{Cuestionario inicial-final}

Se presenta el cuestionario que realizó el alumnado antes y después del CIMA, para realizar un diagnóstico de los conocimientos, las lagunas y las dificultades que tenían los estudiantes al inicio de las clases, y luego comparar los resultados con el cuestionario final, para constatar si había habido aprendizajes. El objetivo de este cuestionario es ver la evolución en la adquisición de contenidos de la materia impartida, y la evolución de los modelos mentales del alumnado. Para el análisis de estos cuestionarios se aplicó una metodología cualitativa de análisis de las respuestas a las preguntas planteadas, el cual nos ha dado la opción de ajustar los contenidos a impartir. Se adjunta el cuestionario en la Tabla 2:

Tabla 2. Cuestionario inicial y final

Cuéntanoslo...

Piensa en una persona cercana a ti que consideres que es mayor

Dinos tu Edad

Dinos tu sexo

No queremos saber quién eres.... pero como el cuestionario se realizará dos veces, necesitamos que te identifiques de alguna manera: las iniciales de tus apellidos, tu DNI sin letra, un mote... como quieras, pero debes hacerlo igual las dos veces, no olvides que opción eliges.

Como te hemos dicho al principio: Piensa en una persona cercana a ti que consideres que es mayor. ¿Cuándo te diste cuenta de que estaba mayor, en qué lo notaste? Explícanoslo

Sigue pensando en esa persona... y ahora compárala con alguien que también consideres que es mayor, pero del otro sexo ¿Están igual? Cuáles son las diferencias o similitudes. Explícanoslas.

Sigue pensando en esa persona... ¿Se queja de las mismas molestias, enfermedades... que tú? Sea cual sea tu respuesta, explícanosla.

Sigue pensando en esa persona... ¿Cómo sabes cuándo está enfermo@? ¿Por qué? ¿Conoces alguna manera de evaluarlo? ¿Cuáles?

Sigue pensando en esa persona... ¿Conoces alguna herramienta de evaluación específica para esa persona mayor? Defínenosla: qué característica tienes, quienes participan, cuándo se hace...

Ciclos de Mejora en el Aula (2020). Experiencias de Innovación Docente de la US Esta obra se distribuye con la licencia Creative Commons 


\section{Aplicación del CIMA}

En esta sección se presenta un relato resumido de las sesiones y la evaluación del aprendizaje de los estudiantes, comparando sus conocimientos iniciales y finales. Se muestra la escalera de aprendizaje de dos de las preguntas del cuestionario para ser pragmáticos y por cuestiones de espacio, y una tabla donde se muestra la evolución individual de cada estudiante.

\section{Relato resumido de las sesiones}

El CIMA se he realizado en 4 sesiones de 2 horas cada, en total 8 horas. Las primeras seis horas se han realizado de manera híbrida, la mitad del alumnado en clase y la otra mitad en casa. Las dos últimas horas del final del CIMA se han realizado online, debido a la declaración del actual estado de alarma decretado el día 9 de noviembre. Inicialmente estaba planteado que las 6 primeras horas fuesen con metodología híbrida y las 2 últimas horas fueran presenciales en grupos pequeños.

La primera actividad fue presentarles el CIMA, explicándoles que formaba parte de una actividad de un curso que estaba realizando a través de ICE y que el objetivo era mejorar las clases y que me gustaría contar con su participación. Se mostraron expectantes. Querían saber, sobre todo, cómo les afectaba a la evaluación.

La segunda actividad fue realizar el cuestionario inicial, y tras finalizar la clase categoricé y analicé sus respuestas para tener una mayor información sobre sus necesidades y conocimientos de partida.

En las siguientes clases fuimos desarrollando las actividades como estaban descritas, siguiendo la secuencia

Ciclos de Mejora en el Aula (2020). Experiencias de Innovación Docente de la US Esta obra se distribuye con la licencia Creative Commons 
de actividades y con el modelo metodológico planteado. Esta secuencia me ayudó a guiar mi docencia y trataba de ceñirme al tiempo. Y esto fue lo primero que observé; era difícil respetar los tiempos, tenía que ser flexible. En general, cuando daban sus opiniones se generaba un clima de confianza y participaban, especialmente los que estaban en clase, y tenía que gestionar de forma más estricta el tiempo. Sin embargo, cuando las actividades implicaban construir un discurso tardaban más y les costaba más trabajo participar. Esta era la situación con el alumnado que estaba presente en el aula, quienes seguían la clase de forma virtual participaban menos en este tipo de actividades.

Terminamos las clases y al grupo que está en clase les pregunto si esta manera de trabajar conjuntamente entre todos les gusta más. Y para mi sorpresa encuentro que con otras palabras me dicen que prefieren mis clases. Es decir, ser pasivos y escuchar "mi speach". Me sorprendió. También los hubo que dijeron que este estilo les gustaba más, pero que no sabían si esto les iba ayudar más o menos para el examen. Encontrándome una vez más con ese pensamiento velado de: "esto cae en el examen".

Otra dificultad que encontré fue que, las últimas clases del CIMA como ya he comentado han sido online. En grupo pequeño, pero todos en casa. Con esta modalidad tardaron más tiempo del que tenía planificado para hacer el caso clínico. Así que al final de la clase no pudieron presentarlo. El objetivo era la corrección entre todos. Algo que he sacado en claro es que trabajar de manera conjunta online es más dificultoso que de manera presencial, Así que los tiempos deben ser más amplios.

Ciclos de Mejora en el Aula (2020). Experiencias de Innovación Docente de la US Esta obra se distribuye con la licencia Creative Commons 


\section{Evaluación del aprendizaje de los estudiantes}

Se realizaron 5 escaleras de conocimiento, una por cada pregunta. Se presentan los resultados de la pregunta 1 y 5 , por ser estos los más significativos. Cada peldaño de la escalera representa un nivel de conocimiento (cuatro en total). En cada peldaño de la escalera, para cada cuestionario (antes y después del CIMA), se presenta el porcentaje de alumnos que se encuentran en dicho nivel de conocimiento.

En el análisis de los resultados de la pregunta 1 (Figura 3) podemos observar que en el cuestionario inicial todo el alumnado se encontraba en los dos primeros escalones de conocimiento, es decir, cuando les preguntábamos sobre las características del envejecimiento sólo se centraban en la apariencia física y algunos incluían alguna característica mental (cognitiva o afectiva). Se encontraron dos obstáculos principales. El primero se sitúa entre el nivel B y el C, y es la falta de inclusión de las características sociales del Envejecimiento. El segundo obstáculo se encuentra entre el nivel $C$ y el $D$ y hace referencia al uso de un lenguaje científico propio de la asignatura.

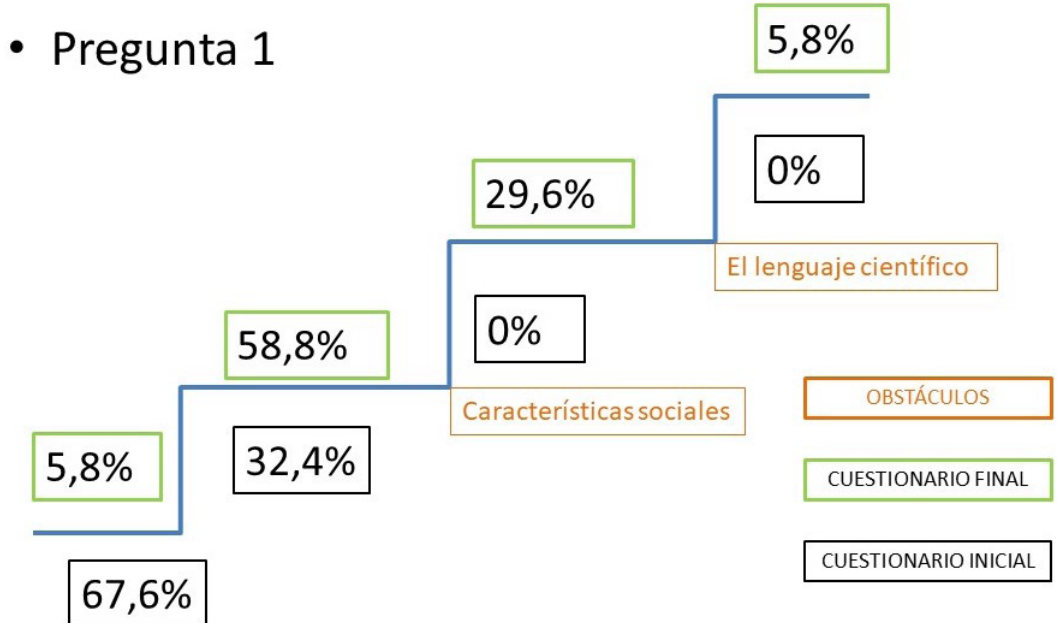

Figura 3. Escalera de aprendizaje para la pregunta 1. Características del Envejecimiento

Ciclos de Mejora en el Aula (2020). Experiencias de Innovación Docente de la US Esta obra se distribuye con la licencia Creative Commons 
El análisis de los resultados de la pregunta 5 (Figura 4) evidencian que los estudiantes han avanzado más con relación a este tema En el cuestionario inicial más del 50\% se encontraba en el escalón más bajo y en el cuestionario final es la que mejores resultados ha obtenido. Se encontró un obstáculo principalmente, el de reconocer la aplicación propia de la Valoración Geriátrica Integral (VGI).

Todo el alumnado supo responder bastante bien a distintas herramientas de evaluación para las diferentes áreas de valoración. En esta pregunta, en el cuestionario final, sí se llegó al nivel máximo y nadie estaba en el escalón más básico.

\section{- Pregunta 5}

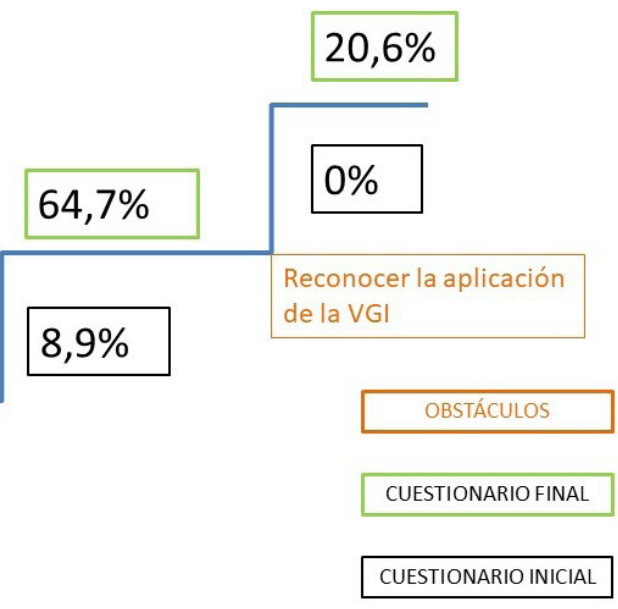

$52,9 \%$

Figura 4. Escalera de aprendizaje para la pregunta 5. Herramientas. VGI.

Continuamos con la presentación del aprendizaje individual de los 34 estudiantes (Tabla3).

En la pregunta 1 se observa, tras el cuestionario final, que más de un tercio del total (frente al 0\% tras el cuestionario inicial) incluyó, además de las características físicas y mentales, las características sociales del envejecimiento y lo hicieron con un lenguaje científico adecuado).

Ciclos de Mejora en el Aula (2020). Experiencias de Innovación Docente de la US Esta obra se distribuye con la licencia Creative Commons 
En la pregunta 2 se observa que, tras el cuestionario final, casi el $60 \%$ del alumnado hablaba del envejecimiento fisiológico diferenciado entre hombres y mujeres, frente a tan sólo el 2,9\% que lo hacía tras el cuestionario inicial.

En la pregunta 3 se observa sin embargo que, tras las actividades realizadas ningún alumno se encontraba en el cuarto escalón de la escalera que ejemplifica la progresión de conocimiento. Esto significa que ningún alumno asoció la pregunta a las características propias de las enfermedades geriátricas. Creemos que esto se debe a la utilización del mismo cuestionario inicial y final. Esto nos lo anotamos como área de mejora para el próximo CIMA.

En la pregunta 4 se observa que, tras el cuestionario final, más de un tercio del alumnado aplicó los modelos de enfermería (y casi el 15\%, además, con un lenguaje científico), frente al 11,8\% inicial.

Finalmente, en la quinta pregunta, se observa que, tras el cuestionario final, casi dos tercios del alumnado conocía más de tres herramientas de valoración (y más del 20\%, además, reconoció la aplicación de la VGI), frente al 8,9\% tras el cuestionario inicial.

Tabla 3. Crecimiento individual de los estudiantes

\begin{tabular}{|c|cccccccc|cc|c|c|}
\hline \multirow{2}{*}{ Estudiantes } & Pregunta 1 & Pregunta 2 & Pregunta 3 & \multicolumn{2}{c|}{ Pregunta 4 } & \multicolumn{2}{c|}{ Pregunta 5 } \\
\cline { 2 - 11 } & I & F & I & F & I & F & I & F & I & F \\
\hline 1 & a & b & b & b & b & c & a & b & a & c \\
2 & a & b & b & b & a & b & a & b & a & c \\
3 & b & c & b & c & a & b & a & b & a & b \\
4 & a & a & b & c & a & b & b & d & b & c \\
5 & a & b & b & c & a & b & b & c & a & c \\
6 & a & c & b & b & c & c & b & c & a & c \\
\hline
\end{tabular}

Ciclos de Mejora en el Aula (2020). Experiencias de Innovación Docente de la US Esta obra se distribuye con la licencia Creative Commons 


\section{Evaluación del CIMA}

En cuanto al aprendizaje del alumnado, es importante destacar que yo suelo interaccionar bastante con ellos. No creo que deba hacerlo de otra manera, pero después de este curso, si hay algo con lo que me quedo es con "dar clases con la boca cerrada" (Finkel, 2008), dejándole a ellos más espacio y margen de actuación.

En cuenta a la metodología, lo que más me ha costado y a la vez más me ha ayudado es la realización del mapa de contenido, ya que con él pude ir de lo particular a lo general, conectando un concepto con otro. Creo que es una fantástica herramienta que ayuda mucho a reflexionar y a situar la docencia en los temas fundamentales. Además, el trabajar con imágenes y artículos previamente seleccionados con un fin, creo que ayuda mucho a afianzar las ideas más importantes. Y se puede relacionar la teoría y la práctica.

En cuanto a la evaluación, ese es mi próximo reto. Creo es de sentido común. Si cambiamos la metodología, la evaluación no puede permanecer igual. El aprendizaje no es sólo memorizar mecánicamente, es comprender y analizar. Y así serán mis próximas evaluaciones.

Una cosa muy importante con la que me quedo: como diría Bain (2007), voy a comenzar mis clases con una pregunta que les haga situarse ante el problema que se busca resolver, porque no se puede dar una respuesta a una pregunta que el alumno no se haya planteado previamente.

Y, por último, pero no menos importante, continuaré con mi diario del profesor (en mi caso, profesora) es algo que me ha hecho reflexionar mucho y no dar por hecho ciertas cosas, per se. La redacción del mismo me ha hecho tomar conciencia diaria y me ha ayudado a tomar decisiones fundamentadas.

Ciclos de Mejora en el Aula (2020). Experiencias de Innovación Docente de la US Esta obra se distribuye con la licencia Creative Commons 
Palabras claves: Enfermería del Envejecimiento; Grado en Enfermería; Docencia Universitaria, Experimentación Docente Universitaria.

Keywords: Geriatric Nursing; Education, Nursing; University Teaching, University Teaching Experimentation.

\section{Referencias Bibliográficas}

Bain, K. (2007). Lo que hacen los mejores profesores universitarios. Valencia: Publicaciones Universidad de valencia.

De Diego, R. (2020). Trabajando la Enfermería Transcultural en el Aula. En E. Navarro y R. Porlán (Coord.), Ciclos de mejora en el aula. Año 2019. Experiencias de innovación docente de la Universidad de Sevilla, (pp. 161-176). Sevilla: Editorial de la Universidad de Sevilla.

Finkel, D. (2008). Dar clases con la boca cerrada. Valencia: Publicaciones Universidad de Valencia.

García, E., Porlán, R. y Navarro, E. (2017). Los fines y los contenidos de ensenanza. En R. Porlán (Coord.), Enseñanza universitaria. Cómo mejorarla (pp. 56-72). Madrid: Ediciones Morata.

Guerra-Martín, D. (2017). Ciclo de Mejora en la asignatura Bioética Fundamental y Clínica en Ciencias de la Salud. En R. Porlán (Coord.), Enseñanza universitaria. Cómo mejorarla (pp. 133-143). Madrid: Ediciones Morata.

Gureckis, T.M. y Markant D.B. (2012). Self-Directed Learning: a cognitive and Computational Perspective. Perspectives on Psychological Science, 7(5), 464-481. Recuperado de: https://doi.org/10.1177/1745691612454304

Pérez, J., Abellán, A., Aceituno, P., y Ramiro, D. (2020). Un perfil de las personas mayores en España, 2020. Indicadores estadísticos básicos. Informes Envejecimiento en red, 25(39). Recuperado de: http://envejecimiento.csic. es/documentos/documentos/enred-indicadoresbasicos2020.pdf>

Porlán, R. (Coord.). (2017). Enseñanza universitaria. Cómo mejorarla. Madrid: Ediciones Morata.

Rodríguez, J.R., Zas, V., Silva, E., Sanchoyerto, R. y Cervantes, M.C. (2014). Evaluación geriátrica integral, importancia,

Ciclos de Mejora en el Aula (2020). Experiencias de Innovación Docente de la US Esta obra se distribuye con la licencia Creative Commons 
ventajas y beneficios en el manejo del adulto mayor. Panorama Cuba y Salud, 9(1), 35-41.

Romero-Serrano, R. (2020). Proyecto docente de Enfermería del Envejecimiento. Curso 2020-21. Centro Universitario de Enfermería San Juan de Dios. Recuperado el 20 de noviembre de 2020 de: http://www.cue.sjd. es/documentos/00-galerias/proyecto docente CUESID 20019-20/PROYECTO DOCENTE 2020-21/3 grado/Proyecto EDE 2020-21.pdf

Tourón, J. y Santiago, R. (2015). El modelo Flipped Learning y el desarrollo del talento en la escuela. Revista de Educación, 368, 196-231.

úbeda, I. (2009). Calidad de vida de los cuidadores familiares: evaluación mediante un doctorado (Tesis Doctoral inédita). Escuela de enfermería de la Universidad de Barcelona. Barcelona. Recuperado de: http://www. tdx.cat/bitstream/handle/10803/2081/IUB TESIS.pdf;jsessionid=8EB7300C3D7780DB4CC1575BE6095C1B. tdx2? sequence $=1$

Vallejo, J.M., Rodríguez, M. y Valverde, M.M. (2007). Valoración Geriátrica. Un modelo de registro en residencias de ancianOs. GEROKOMOS, 18(2), 72-76.

Ciclos de Mejora en el Aula (2020). Experiencias de Innovación Docente de la US Esta obra se distribuye con la licencia Creative Commons 